\title{
Perianal Plasmablastic Lymphoma Masquerading as a Buschke-Löwenstein- like Tumour in an HIV-infected Patient with Recurrent Anal Condyloma
}

\author{
Xavier Rivera Rivera, Joshua Baalwa \\ Department of Pathology and Laboratory Medicine, The University of Texas Health Sciences Center at Houston, McGovern Medical School, Houston, Texas, USA
}

Received: 08/04/2021

Accepted: $25 / 05 / 2021$

Published: $11 / 06 / 2021$

\begin{abstract}
How to cite this article: Rivera Rivera X, Baalwa J. Perianal plasmablastic lymphoma masquerading as Buschke-Löwenstein like tumour in an HIV-infected patient with recurrent anal condyloma. EJCRIM 2021;8: doi:10.12890/2021_002552.
\end{abstract}

Conflicts of Interests: The authors declare there are no competing interests.

Acknowledgements: We thank Dr Stefanos Millas for the clinical photographs.

This article is licensed under a Commons Attribution Non-Commercial 4.0 License

\section{ABSTRACT}

Plasmablastic lymphoma (PBL) commonly presents as a primary (de novo) oral or extraoral mucocutaneous or nodal mass lesion in patients with HIV/AIDS. PBL developing as a secondary malignancy at the same location as a pre-existing tumour is extremely rare and has never been reported in association with longstanding or recurrent anal condyloma. A Buschke-Löwenstein tumour is a rare gigantic, locally destructive condyloma that is usually located in the anogenital region. We report a case of a diagnostically and therapeutically challenging PBL that presented as a rapidly enlarging mass underlying a giant condyloma, thereby mimicking a benign Buschke-Löwenstein tumour. Clinical suspicion was further masked by the co-presence of fistulae in ano and adjacent abscess pockets at the time of diagnosis. By the time of final diagnosis, the lymphoma had disseminated to regional lymph nodes, a month later to pleural cavities and 4 months later to the leptomeninges and bilateral kidneys, leading to permanent deferral of chemotherapeutic intervention.

\section{LEARNING POINTS}

- Plasmablastic lymphoma presenting as a secondary tumour in a patient with pre-existing giant anal condyloma has not been reported previously in the literature.

- Unusual clinical presentation of a recurrent giant condyloma, especially rapid growth and significant change in physical appearance, should kindle a high index of suspicion for a secondary aggressive tumour.

- Plasmablastic lymphoma disseminates early and rapidly, which complicates its response to treatment.

\section{KEYWORDS}

Plasmablastic lymphoma, Buschke-Löwenstein like tumor, antiretroviral therapy associated immune reconstitution

\section{CASE DESCRIPTION}

A 34-year-old man with a 4-year history of recurrent perianal and anal condylomata refractory to multiple fulgurations (Fig. 1) and complicated by recurrent fistulae in ano and abscess formation, was referred to the surgery clinic due to a rapidly enlarging, excruciatingly painful perianal mass temporarily relieved by analgesics. He had a past medical history of HIV/AIDS since 2009 that had over the years been complicated by multiple opportunistic infections including cryptococcal meningitis, syphilis, tuberculosis and Kaposi's sarcoma, mainly attributable to poor compliance with antiretroviral therapy. On examination, he was found to have a large fungating right perianal mass associated with multiple draining fistulae and an adjacent abscess, laterally (Fig. 2). Ano-perineal computerized tomography showed a $4.8 \times 11.2 \times 6.3 \mathrm{~cm}$ hyperdense enhancing mass in the right perineum. The patient underwent drainage of the abscess and multiple incision 
biopsies of the mass were taken. Surprisingly, the biopsies revealed a final diagnosis of plasmablastic lymphoma (PBL) underlying a giant perianal condyloma (Figs. 3 and 4).

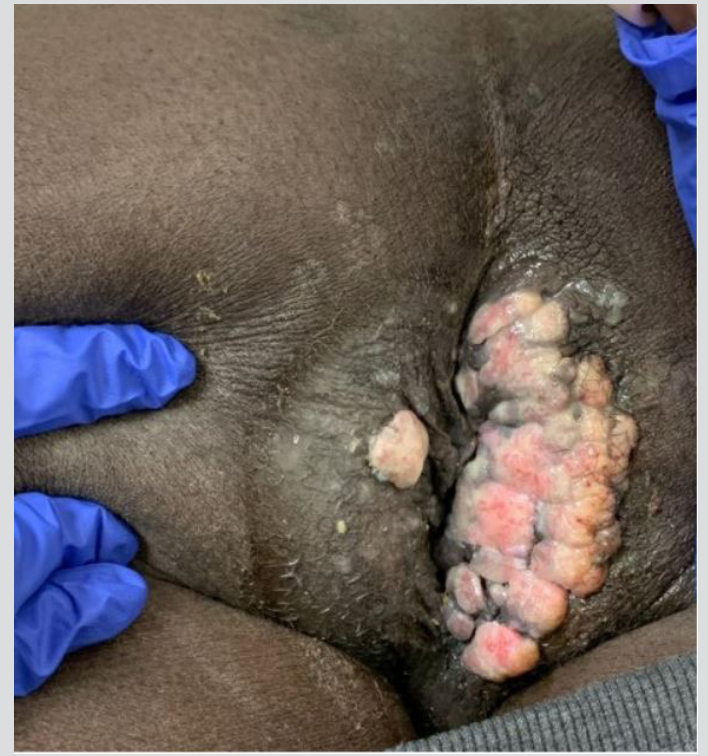

Figure 1. Perianal condyloma shown here in July 2020 after previously recurring several times despite multiple fulgurations

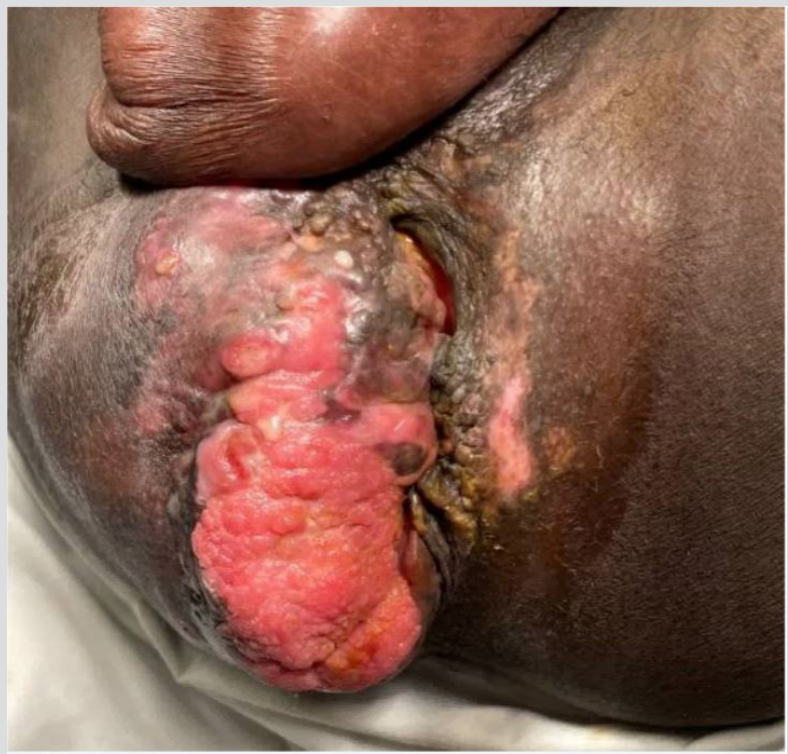

Figure 2. Condyloma presenting as a disfiguring fungating mass in November 2020 thus mimicking a Buschke-Löwenstein tumor. An associated abscess was found to be the cause of induration lateral to the fungating mass. Biopsies of the mass revealed a diagnosis of plasmablastic lymphoma.

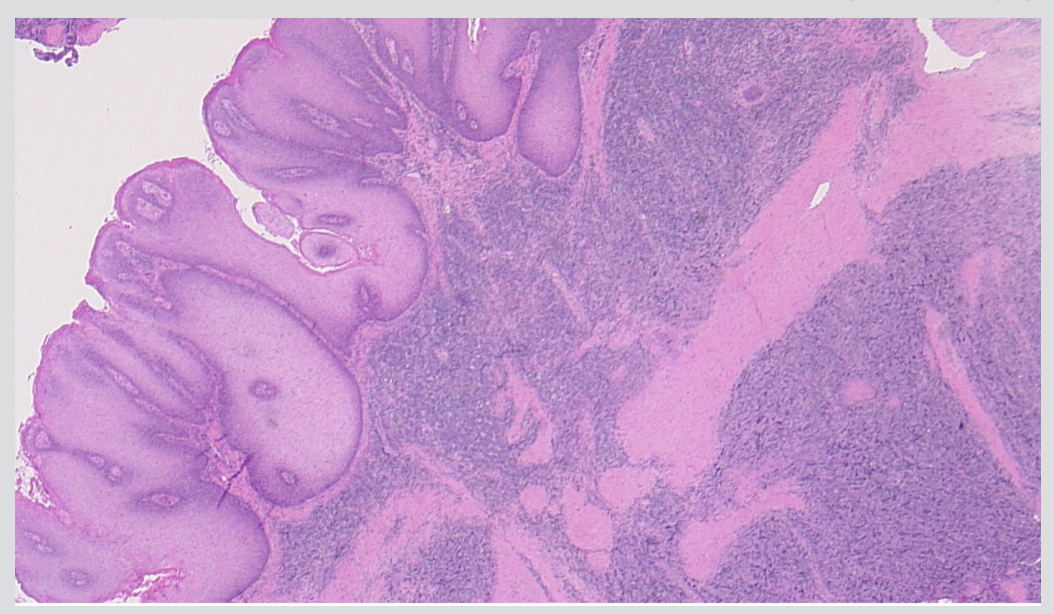

Figure 3. Histology of plasmablastic lymphoma (involving the dermis, right half of image) underlying a giant condyloma (involving the epithelial surface, left half of image).

Staging computerized tomography (CT) demonstrated regional adenopathy and biopsies revealed nodal involvement by the same lymphoma. The patient was commenced on a chemotherapy regimen (etoposide, prednisolone, Oncovin, cyclophosphamide, hydroxydaunorubicin) which was interrupted due to toxicity. A month following the lymphoma diagnosis, he was found to have malignant pleural effusions. Two months later he presented with altered mental state and CT of head showed diffuse enhancing foci throughout the calvarium, skull base and mandible; leptomeningeal enhancement in the bilateral parieto-occipital sulci; subtle enhancing soft tissue components in the parietooccipital and frontal lobes; and a $2 \mathrm{~cm}$ mass in the right mandibular ramus consistent with metastatic spread of the patient's lymphoma. Additional imaging revealed possible bilateral renal involvement by lymphoma. Cytological studies confirmed central nervous system involvement by the same lymphoma. The patient received several doses of Velcade-ifosfamide, carboplatin and etoposide including intrathecal chemotherapy which he poorly tolerated due to neutropenia and mucositis. The chemotherapy regimen was switched to Velcade, methotrexate and cytarabine, however, it was complicated by tumour lysis syndrome. The patient continued to deteriorate, compelling his family's decision to defer any further lymphoma treatment and opt for comfort measures. 

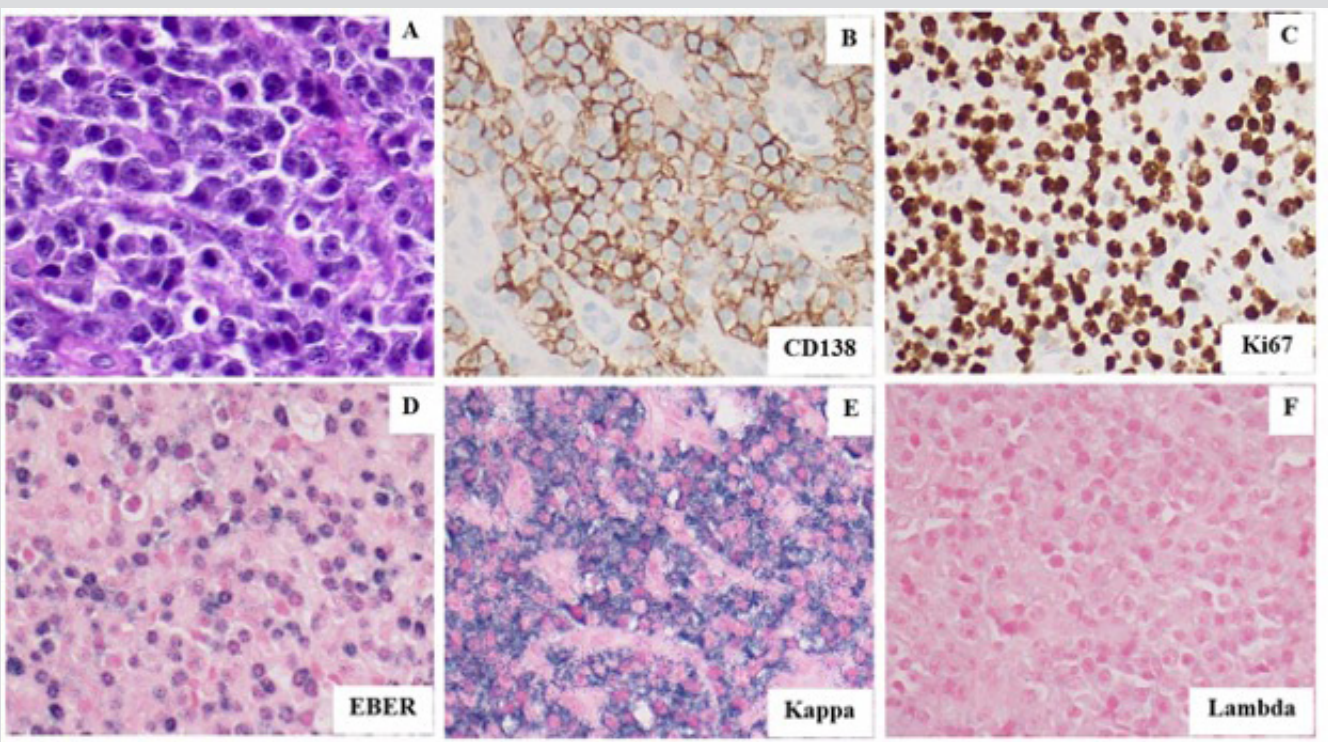

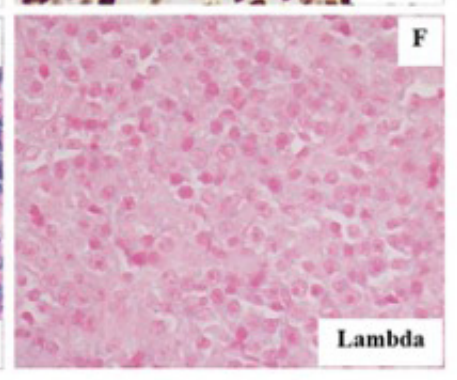

Figure 4. (A). Hematoxylin and Eosin stain shows the tumor to be composed of numerous large plasmablasts. (B). CD138 immunoperoxidase stain confirms the neoplastic cells show plasmacytic differentiation. (C). Ki67 immunoperoxidase stain shows that the neoplastic cells have a high proliferation index consistent with a high-grade malignancy. (D). Epstein Barr Virus Encoded RNA (EBER) in situ hybridization stain shows the neoplastic cells to be diffusely positive for Epstein Barr Virus. (E-F). The neoplastic cells show strong kappa light chain restriction by in situ RNA hybridization staining supporting their clonal nature. All images taken at $400 \mathrm{X}$ magnification.

\section{DISCUSSION}

Extraoral PBL often presents as a primary mucocutaneous or nodal tumour ${ }^{[1]}$. Among extraoral locations, the anal locoregion is a more common site of presentation than others ${ }^{[2]}$ where it may be associated with fistulae or abscesses. However, extremely uncommon or to our knowledge not yet reported, is concurrent presentation of PBL as a secondary neoplasm underlying a primary anal tumour. Here we report an unusual presentation of PBL concurrently presenting beneath a pre-existing giant condyloma thereby masquerading as a benign tumour and thus evading early clinical suspicion. Likewise, immediately prior to diagnosis, the patient reported rapid enlargement of his anal condyloma, however, because similar prior presentations of his condyloma had revealed fistulae, abscess formation and no malignancy, the possibility of an aggressive new malignancy was initially not suspected.

Of note, our patient's condyloma-PBL mass showed unusual clinical features in the days leading to lymphoma diagnosis. In July 2020 , prior to fulguration, the condyloma had a typical warty appearance. However, between October and November 2020, the time period leading to lymphoma diagnosis, the condyloma presented as a rapidly enlarging, excruciatingly painful indurated mass with a fungating-like appearance, thus mimicking a Buschke-Löwenstein tumour. A characteristic feature of Buschke-Löwenstein tumours is their locally destructive nature with a relative low risk of malignant transformation ${ }^{[3]}$. However, Buschke-Löwenstein tumours usually evolve insidiously and retain a typical warty appearance. Therefore, this case further shows the value of an in-depth analysis of differences in clinical presentation as an aid to early clinical suspicion of malignancy. Early clinical suspicion of an aggressive malignancy is important because it informs appropriate scheduling of clinical visits, any indicated surgical interventions and guides timely pathological work-up.

$\mathrm{PBL}$ is known to disseminate early and is therefore associated with a dismal prognosis, with a median overall survival averaging 1 year ${ }^{[1,4]}$. It is however reported that HIV patients optimally treated with HAART may have improved overall survival ${ }^{[5]}$. Likewise, recently it has been reported that early diagnosis, and therefore early intervention with chemotherapy, may improve median overall survival by several months $^{[6]}$. Our patient was unfortunate to have had disseminated disease by the time of diagnosis, however, at that time he had an undetectable viral load and an estimated absolute CD4 count $>300$. Thus, it is intriguing that his illness rapidly progressed to the point when it was determined there was no further benefit from chemotherapy, 4 months after diagnosis. Notably, 3 months prior to his lymphoma diagnosis, the patient achieved the first ever undetectable HIV viral load in 10 years which at that time corresponded to a $>2$ log reduction in viral load (Fig. 5). Therefore, according to Gopal et al., his lymphoma met the criteria for lymphoma immune reconstitution inflammatory syndrome, which was shown by the same authors to show relatively poor overall survival when compared with non-immune reconstitution inflammatory syndrome lymphoma ${ }^{[7]}$. Whether immune reconstitution inflammatory syndrome might explain our patient's rapid evolution and dissemination of his lymphoma remains unclear. 
2016: Patient is diagnosed with anal condyloma for the first time

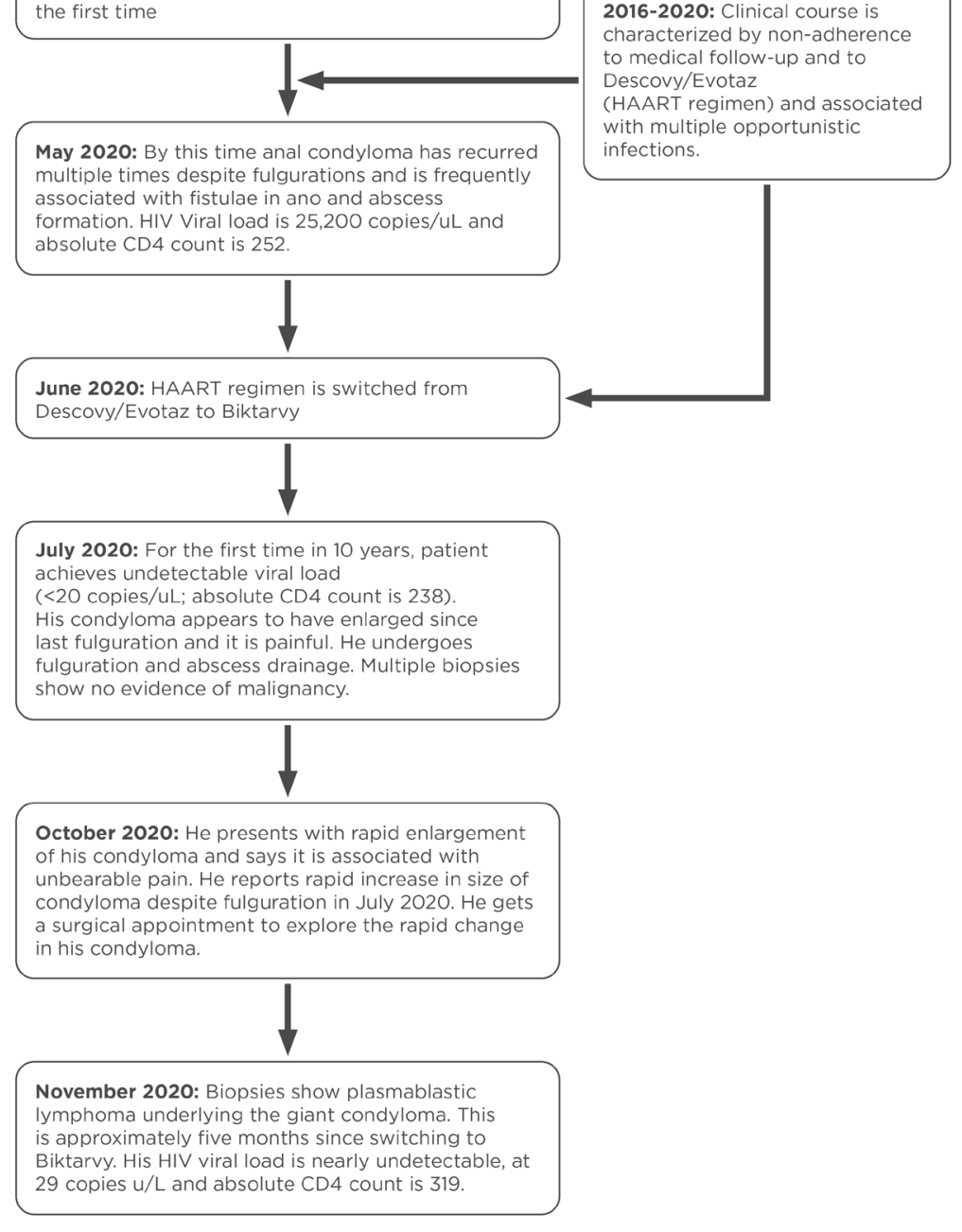

Figure 5. A timeline summary of progression from recurrent condylomas to development of giant condyloma associated with underlying lymphoma

\section{CONCLUSION}

Our case further emphasizes the need to maintain a high index of clinical suspicion for easy-to-overlook secondary malignancies which may evolve at the same location as pre-existing benign primary tumours, and for unmasking of malignancies following immune reconstitution in HIV/AIDS patients.

\section{REFERENCES}

1. Castillo JJ, Bibas M, Miranda RN. The biology and treatment of plasmablastic lymphoma. Blood 2015;125(15):2323-2330.

2. Meer S, Perner Y, McAlpine ED, Willem P. Extraoral plasmablastic lymphomas in a high human immunodeficiency virus endemic area. Histopathology 2020;76:212-221.

3. Creasman C, Haas PA, Fox Jr TA, Balazs M. Malignant transformation of anorectal giant condyloma acuminatum (Buschke-Loewenstein tumor). Dis Colon Rectum 1989;32(6):481-487.

4. Lopez A, Abrisqueta P. Plasmablastic lymphoma: current perspectives. Blood Lymphat Cancer 2018;8:63-70.

5. Noy A, Lensing SY, Moore PC, Gupta N, Aboulafia D, Ambinder R, et al. Plasmablastic lymphoma is treatable in the HAART era. A 10 year retrospective by the AIDS Malignancy Consortium. Leuk Lymphoma 2016:57(7):1731-1734.

6. Tchernonog E, Faurie P, Coppo P, Monjanel H, Bonnet A, Algarte Génin M, et al. Clinical characteristics and prognostic factors of plasmablastic lymphoma patients: analysis of 135 patients from the LYSA group. Ann Oncol 2017;28(4):843-848.

7. Gopal S, Patel MR, Achenbach CJ, Yanik EL, Cole SR, Napravnik S, et al. Lymphoma immune reconstitution inflammatory syndrome in the center for AIDS research network of integrated clinical systems cohort. Clin Infect Dis 2014;59(2):279-286. 